\title{
Exact Multi-Matrix Correlators
}

\author{
Rajsekhar Bhattacharyya ${ }^{1}$, Storm Collins ${ }^{2}$ and Robert de Mello Koch ${ }^{2,3}$ \\ 1 Department of Physics, \\ Dinabandhu Andrews College, \\ Kolkata-700084, \\ India \\ 2 National Institute for Theoretical Physics, \\ Department of Physics and Centre for Theoretical Physics, \\ University of the Witwatersrand, \\ Wits, 2050, \\ South Africa \\ ${ }^{3}$ Stellenbosch Institute for Advanced Studies, \\ Stellenbosch, \\ South Africa \\ E-mail: rajsekhar@dacollege.org, Storm.Collins@students.wits.ac.za, \\ robert@neo.phys.wits.ac.za
}

\begin{abstract}
We argue that restricted Schur polynomials provide a useful parameterization of the complete set of gauge invariant variables of multi-matrix models. The two point functions of restricted Schur polynomials are evaluated exactly in the free field theory limit. They have diagonal two point functions.
\end{abstract}

Keywords: Giant Gravitons, AdS/CFT correspondence, super Yang-Mills theory. 


\section{Contents}

1. Introduction $\quad 1$

2. Two Matrix Model 2

3. Counting 5

4. Examples $\quad 6$

5. Generalization to Multi-Matrix Models $\quad 9$

$\begin{array}{ll}\text { 6. Numerical Tests } & 10\end{array}$

$\begin{array}{ll}\text { 7. Conclusions } & 11\end{array}$

\section{Introduction}

The Maldacena conjecture[1], which claims an equivalence between $\mathcal{N}=4$ super YangMills theory and type IIB string theory in the $\mathrm{AdS}_{5} \times \mathrm{S}^{5}$ background, is a significant step in establishing the expectation that large $N$ gauge theories are equivalent to string theory. One approach towards establishing the conjecture is to implement a direct change of variables from the matrices of the gauge theory to the fields of string theory. Collective field theory[2] provides a clear and well defined scheme for making this transition. The first step in this approach is to find a useful parameterization of the complete set of gauge invariant variables of the matrix model. For a model with more than one matrix, this purely kinematical problem is already nontrivial. In this note, we call this the kinematical problem.

The $\mathcal{N}=4$ super Yang-Mills theory has six hermittian Higgs fields, $\phi_{i} i=1,2, \ldots, 6$, transforming in the adjoint of $U(N)$. Form the complex combinations $Z=\phi_{1}+i \phi_{2}$, $X=\phi_{3}+i \phi_{4}$ and $Y=\phi_{5}+i \phi_{6}$. The space of $\frac{1}{2}$ BPS representations in $\mathcal{N}=4$ super Yang-Mills theory are in one-to-one correspondence with the Schur polynomials built out of $Z$ [3]. These Schur polynomials have diagonal two point functions[3]. Using insights from the dual quantum gravity, excitations of these $\frac{1}{2}$ BPS states, restricted 
Schur polynomials, have been identified[4]. The restricted Schur polynomial is obtained by "attaching" open string words $W$ to the Schur polynomial. The letters of these open string words can be fermions, gauge fields or any of the six Higgs fields. If the word $W$ is to be dual to an open string, it should contain $O(\sqrt{N})$ letters. If the restricted Schur polynomial contains $O(N)$ fields, it is dual to a membrane with open strings attached; if it contains $O\left(N^{2}\right)$ fields, it is dual to a string moving in a new geometry. Thus, the restricted Schur polynomial starts to address the kinematical problem outlined above. The technology for computing correlators of restricted Schur polynomials has enjoyed some progress[5],[6],[7]. For related work see[8].

In two recent papers, a large class of operators that diagonalize the two point functions in the free field theory limit have been given[9],[10]. These include operators built from $Z$ and $Z^{\dagger}[9]$ and operators built from $X, Y$ and $Z[10]$. Further, the number of such operators matches the number of gauge invariant operators that can be constructed. The results of [9],[10] therefore solve the kinematical problem, in the Higgs sector. This basis also gives a group theoretic way to approach higher point functions (see [10] where three and higher point functions are obtained) and to obtain factorization equations which can be used to build a probability interpretation[11]. By exploiting supergroups [10] have also explained how to include fermions in addition to the Higgs fields. Finally, the one loop correction to these two points functions has been considered in [12].

The purpose of this communication is to argue that the restricted Schur polynomials themselves, provide a solution to the kinematical problem, in the Higgs sector. This is not unexpected. Indeed, if one excites a $\frac{1}{2}$ BPS state by attaching a large number of words that are composed of a single letter, one is building up multi-matrix operators. Our argument is simple, employing only very basic group theory. Further, by exploiting the technology already available for restricted Schur polynomials, explicit formulas for the relevant restricted Schur polynomials and their two point functions are easily obtained.

\section{Two Matrix Model}

Consider a $d=0$ matrix model with two types of complex matrices $A$ and $B^{1}$. These complex matrices act on an $N$-dimensional vector space $V, A: V \rightarrow V$. The non-zero correlators are

$$
\left\langle(A)_{j}^{i}\left(A^{\dagger}\right)_{l}^{k}\right\rangle=\delta_{l}^{i} \delta_{j}^{k}=\left\langle(B)_{j}^{i}\left(B^{\dagger}\right)_{l}^{k}\right\rangle
$$

Consider the operators

$$
\chi_{\alpha}=\operatorname{Tr}_{n+m}\left(O_{\alpha} A^{\otimes n} \otimes B^{\otimes m}\right),
$$

\footnotetext{
${ }^{1}$ The spacetime dependence which has been dropped from this model can be trivially reinstated using the conformal symmetry of the super Yang-Mills theory.
} 
where $\operatorname{Tr}_{n+m}$ is a trace over $V^{\otimes(n+m)} . \quad A^{\otimes n} \otimes B^{\otimes m}$ is a shorthand for the tensor $A_{j_{1}}^{i_{1}} A_{j_{2}}^{i_{2}} \cdots A_{j_{n}}^{i_{n}} B_{j_{n+1}}^{i_{n+1}} B_{j_{n+2}}^{i_{n+2}} \cdots B_{j_{n+m}}^{i_{n+m}}$ and

$$
\operatorname{Tr}_{n+m}\left(O_{\alpha} A^{\otimes n} \otimes B^{\otimes m}\right)=\left(O_{\alpha}\right)_{i_{1} i_{2} \cdots i_{n+m}}^{j_{1} j_{2} \cdots j_{n+m}} A_{j_{1}}^{i_{1}} A_{j_{2}}^{i_{2}} \cdots A_{j_{n}}^{i_{n}} B_{j_{n+1}}^{i_{n+1}} B_{j_{n+2}}^{i_{n+2}} \cdots B_{j_{n+m}}^{i_{n+m}}
$$

We are interested in computing the correlator $\left\langle\chi_{\alpha} \chi_{\beta}^{\dagger}\right\rangle$. Using (2.1) we obtain

$$
\left\langle\chi_{\alpha} \chi_{\beta}^{\dagger}\right\rangle=\sum_{\gamma \in S_{n} \times S_{m}} \operatorname{Tr}_{n+m}\left(O_{\alpha} \gamma O_{\beta}^{\dagger} \gamma^{-1}\right)
$$

The sum over $\gamma$ is a sum over all possible Wick contractions. Assume that

$$
O_{\beta}=\gamma O_{\beta} \gamma^{-1}, \quad n ! m ! \operatorname{Tr}_{n+m}\left(O_{\alpha} O_{\beta}^{\dagger}\right)=\mathcal{N}_{\alpha} \delta_{\alpha \beta}
$$

This means that the $O_{\alpha}$ are symmetric branching operators [9]. Then the operators $\chi_{\alpha}$ diagonalize the two point function

$$
\left\langle\chi_{\alpha} \chi_{\beta}^{\dagger}\right\rangle=\mathcal{N}_{\alpha} \delta_{\alpha \beta}
$$

We will now argue that a complete set of $O_{\alpha}$ are given by

$$
O_{\alpha}=\frac{1}{n ! m !} \sum_{\sigma \in S_{n+m}} \operatorname{Tr}_{R_{\alpha}}\left(\Gamma_{R}(\sigma)\right) \sigma
$$

where $R_{\alpha}$ is an irreducible representation of $S_{n} \times S_{m}$ and $R$ is an irreducible representation of $S_{n+m}$. The $S_{n} \times S_{m}$ subgroup is chosen so that $S_{n}$ acts on the indices of the $A$ s and $S_{m}$ on the indices of the $B$ s. Thus, the $S_{n} \times S_{m}$ subgroup that we sum over to include all possible Wick contractions is the same subgroup for which $R_{\alpha}$ is an irreducible representation. Under restricting to the $S_{n} \times S_{m}$ subgroup, $R$ will in general be reducible. We can decompose the carrier space of irreducible representation $R$ according to the irreducible $S_{n} \times S_{m}$ representations that are subduced. $\operatorname{Tr}_{R_{\alpha}}$ is an instruction to trace only over the subspace corresponding to $R_{\alpha}$. For more details see [5]. In this case, the $\chi_{\alpha}$ are nothing but restricted Schur polynomials, so that the restricted Schur polynomials solve the kinematical problem and have diagonal two point functions.

Demonstration that $O_{\beta}=\gamma O_{\beta} \gamma^{-1}$ :

$$
\begin{aligned}
\gamma O_{\alpha} \gamma^{-1} & =\frac{1}{n ! m !} \sum_{\sigma \in S_{n+m}} \operatorname{Tr}_{R_{\alpha}}\left(\Gamma_{R}(\sigma)\right) \gamma \sigma \gamma^{-1}=\frac{1}{n ! m !} \sum_{\tau \in S_{n+m}} \operatorname{Tr}_{R_{\alpha}}\left(\Gamma_{R}\left(\gamma^{-1} \tau \gamma\right)\right) \tau \\
& =\frac{1}{n ! m !} \sum_{\tau \in S_{n+m}} \operatorname{Tr}_{R_{\alpha}}\left(\Gamma_{R}\left(\gamma^{-1}\right) \Gamma_{R}(\tau) \Gamma_{R}(\gamma)\right) \tau
\end{aligned}
$$




$$
\begin{aligned}
& =\frac{1}{n ! m !} \sum_{\tau \in S_{n+m}} \operatorname{Tr}_{R_{\alpha}}\left(\Gamma_{R_{\alpha}}\left(\gamma^{-1}\right) \Gamma_{R}(\tau) \Gamma_{R_{\alpha}}(\gamma)\right) \tau \\
& =\frac{1}{n ! m !} \sum_{\tau \in S_{n+m}} \operatorname{Tr}_{R_{\alpha}}\left(\Gamma_{R}(\tau)\right) \tau=O_{\alpha} .
\end{aligned}
$$

We used the fact that $\gamma \in S_{n} \times S_{m}$, that $R_{\alpha}$ is an irreducible representation of $S_{n} \times S_{m}$ and that the trace is invariant under a similarity transformation.

Demonstration that $\operatorname{Tr}_{n+m}\left(O_{\alpha} O_{\beta}^{\dagger}\right)=\mathcal{N}_{\alpha} \delta_{\alpha \beta}$ :

$$
\begin{aligned}
n ! m ! \operatorname{Tr}_{n+m}\left(O_{\alpha} O_{\beta}^{\dagger}\right) & =\frac{1}{n ! m !} \sum_{\sigma \in S_{n+m}} \sum_{\tau \in S_{n+m}} \operatorname{Tr}_{R_{\alpha}}\left(\Gamma_{R}(\sigma)\right) \operatorname{Tr}_{S_{\beta}}\left(\Gamma_{S}(\tau)\right)^{*} \operatorname{Tr}_{n+m}\left(\sigma \tau^{-1}\right) \\
& =\frac{1}{n ! m !} \sum_{\sigma \in S_{n+m}} \sum_{\tau \in S_{n+m}} \operatorname{Tr}_{R_{\alpha}}\left(\Gamma_{R}(\sigma)\right) \operatorname{Tr}_{S_{\beta}}\left(\Gamma_{S}(\tau)\right)^{*} N^{C\left(\sigma \tau^{-1}\right)} \\
& =\frac{1}{n ! m !} \sum_{\psi \in S_{n+m}} \sum_{\tau \in S_{n+m}} \operatorname{Tr}_{R_{\alpha}}\left(\Gamma_{R}(\psi \tau)\right) \operatorname{Tr}_{S_{\beta}}\left(\Gamma_{S}(\tau)\right)^{*} N^{C(\psi)} .
\end{aligned}
$$

Now, lets perform the sum over $\tau$ (use the fact that $\left(P_{S \rightarrow S_{\beta}}\right)_{j r}^{*}=\left(P_{S \rightarrow S_{\beta}}\right)_{r j}$ because the projector $P_{S \rightarrow S_{\beta}}$ is hermittian)

$$
\begin{aligned}
& \sum_{\tau \in S_{n+m}} \operatorname{Tr}_{R_{\alpha}}\left(\Gamma_{R}(\psi \tau)\right) \operatorname{Tr}_{S_{\beta}}\left(\Gamma_{S}(\tau)\right)^{*} \\
= & \sum_{\tau \in S_{n+m}} \sum_{i j q r}\left(P_{R \rightarrow R_{\alpha}} \Gamma_{R}(\psi)\right)_{i q}\left(\Gamma_{R}(\tau)\right)_{q i}\left(P_{S \rightarrow S_{\beta}}\right)_{r j}\left(\Gamma_{S}(\tau)\right)_{r j}^{*} \\
= & \delta_{R S} \frac{(n+m) !}{d_{R}} \sum_{i q}\left(P_{R \rightarrow R_{\alpha}} \Gamma_{R}(\psi)\right)_{i q}\left(P_{S \rightarrow S_{\beta}}\right)_{q i}=\delta_{R S} \delta_{R_{\alpha} S_{\beta}} \operatorname{Tr}_{R_{\alpha}}\left(\Gamma_{R}(\psi)\right) \frac{(n+m) !}{d_{R}} .
\end{aligned}
$$

We have used the fundamental orthogonality relation

$$
\sum_{\tau \in S_{n+m}}\left(\Gamma_{R}(\tau)\right)_{q i}\left(\Gamma_{S}(\tau)\right)_{r j}^{*}=\frac{(n+m) !}{d_{R}} \delta_{q r} \delta_{i j} \delta_{R S} .
$$

Thus, using appendix F of [5] we obtain

$$
\begin{aligned}
n ! m ! \operatorname{Tr}\left(O_{\alpha} O_{\beta}^{\dagger}\right) & =\frac{\delta_{R S} \delta_{R_{\alpha} S_{\beta}}}{n ! m !} \frac{(n+m) !}{d_{R}} \sum_{\psi \in S_{n+m}} \operatorname{Tr}_{R_{\alpha}}\left(\Gamma_{R}(\psi)\right) N^{C(\psi)} \\
& =\frac{\delta_{R S} \delta_{R_{\alpha} S_{\beta}}}{n ! m !} \frac{(n+m) !}{d_{R}} d_{R_{\alpha}} f_{R}=\delta_{R S} \delta_{R_{\alpha} S_{\beta}} \frac{(\text { hooks })_{R}}{(\text { hooks })_{R_{\alpha}}} f_{R} .
\end{aligned}
$$

$R_{\alpha}$ is a rep of $S_{n} \times S_{m}$ which is labelled by one Young diagram of $n$ boxes, $R_{n}$, and one Young diagram of $m$ boxes, $R_{m}$. (hooks) $R_{\alpha}$ is the product of (hooks) $R_{n}$ with (hooks) $R_{m}$. 
Arguing as we did above, it is simple to obtain

$$
O_{\alpha} O_{\beta}=\frac{(n+m) !}{d_{R} n ! m !} \delta_{\alpha \beta} O_{\alpha}
$$

Thus, up to normalization our operators $O_{\alpha}$ are projectors. For an earlier use of projectors, along the lines of this note but in the setting of a single matrix, see [13]. From now on we write $\chi_{R, R_{\alpha}}$ instead of $\chi_{\alpha}$. In general, the row and column index of the restriction $R_{\alpha}$ can be different (see [4],[5] for a detailed discussion). Spell out these row and column indices by replacing $R_{\alpha} \rightarrow\left(r_{\alpha_{1}}, r_{\alpha_{2}}\right)$. The two point function is

$$
\left\langle\chi_{R,\left(r_{\alpha_{1}}, r_{\alpha_{2}}\right)} \chi_{S,\left(s_{\beta_{1}}, s_{\beta_{2}}\right)}^{\dagger}\right\rangle=\delta_{R S} \delta_{r_{\alpha_{1}} s_{\beta_{1}}} \delta_{r_{\alpha_{2}} s_{\beta_{2}}} \frac{(\text { hooks })_{R}}{(\text { hooks })_{R_{\alpha}}} f_{R}
$$

It is equally easy to argue that

$$
\left\langle\chi_{R,\left(r_{\alpha_{1}}, r_{\alpha_{2}}\right)} \chi_{S,\left(s_{\beta_{1}}, s_{\beta_{2}}\right)}\right\rangle=\delta_{R S} \delta_{r_{\alpha_{1} s_{\beta_{2}}}} \delta_{r_{\alpha_{2}} s_{\beta_{1}}} \frac{(\text { hooks })_{R}}{(\text { hooks })_{R_{\alpha}}} f_{R}
$$

In sections 3 and 5 we will give evidence that the number of restricted Schur polynomials $\chi_{R, R_{\alpha}}$ is equal to the number of gauge invariant operators in the matrix model.

\section{Counting}

The number of gauge invariant operators $N(n, m)$ built out of $n A$ s and $m B$ s is given by Polya theory as

$$
\prod_{k=1}^{\infty} \frac{1}{1-\left(x^{k}+y^{k}\right)}=\sum_{n, m} N(n, m) x^{n} y^{m} .
$$

We claim that the number of gauge invariant operators $N(n, m)$ is equal to the number

of restricted Schur polynomials $\chi_{R, R_{\alpha}}$ with $R$ an irreducible representation of $S_{n+m}$ and $R_{\alpha}$ an irreducible representation of $S_{n} \times S_{m}$. It is easy to check for small values of $n$ and $m$ that this is indeed the case. As an example, consider $m=n=2$. In this case, $R$ is an irreducible representation of $S_{4}$. We easily find $N(2,2)=10$. The allowed restricted traces $\left(R ; R_{\alpha}\right)$ are

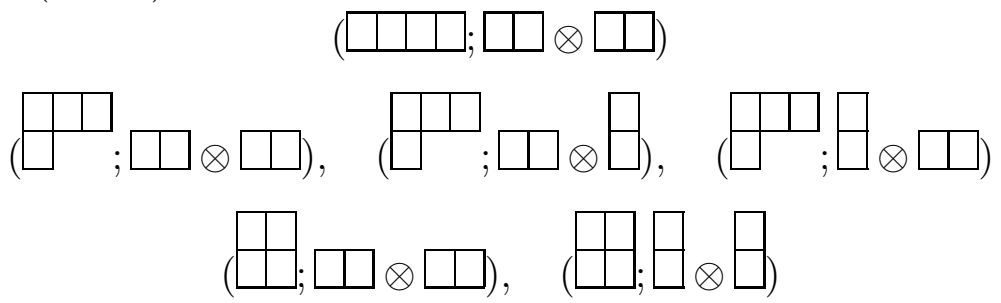




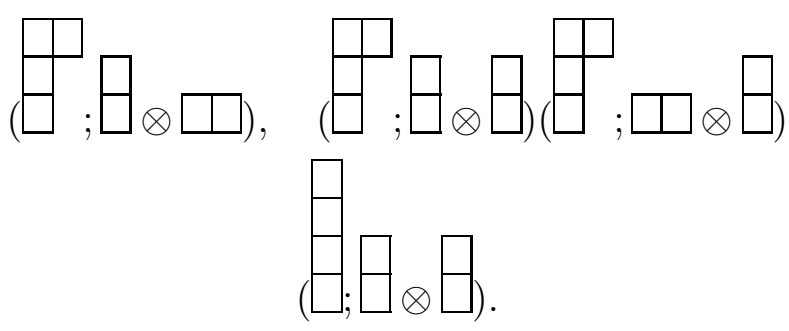

Thus, there are indeed ten possible restricted Schur polynomials.

There is a subtlety that did not show up in the above example: in the notation of [5],[4], we can trace over an off the diagonal block. For example, amoung the $S_{3} \times S_{3}$ irreducible representations subduced by the $S_{6}$ irreducible representation

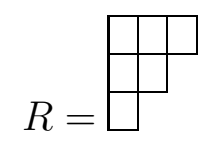

we find two copies of

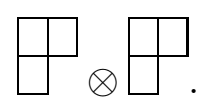

Call these two copies $R_{\alpha}^{(1)}$ and $R_{\alpha}^{(2)}$. When performing the restricted trace, we can use $R_{\alpha}^{(i)}$ for the row index and $R_{\alpha}^{(j)}$ for the column index with $R_{\alpha}^{(i)} \neq R_{\alpha}^{(j)}$. Thus, there are four possible operators we can define. In general, if $R$ subduced $m$ copies of an irreducible representation $R_{\alpha}$ we would be able to construct $m^{2}$ independent operators. For further details consult section 2.2 of [5].

\section{Examples}

The simplest way to construct restricted Schur polynomials, is to use a projection operator to implement the restricted trace. In this section we will construct restricted Schur polynomials built from at most three matrices, which can be any of two different types $X$ or $Y$. This will already allow us to see that the restricted Schur polynomials define a different basis for gauge invariant operators, than the bases given in [9],[10]. The construction of

$$
\chi_{\square ; \square^{\otimes}}=\operatorname{Tr}(X) \operatorname{Tr}(Y)+\operatorname{Tr}(X Y), \quad \chi_{\square ; \square \otimes \square}=\operatorname{Tr}(X) \operatorname{Tr}(Y)-\operatorname{Tr}(X Y),
$$

is particularly simple because we do not need a projector to implement the restricted trace. This follows because $\square^{\otimes} \square$ is the only $S_{1} \times S_{1}$ irreducible representation subduced from either $\square$ or $\square$. Up to normalization, these are identical to the operators constructed in appendix E1 of [10]. Consider next

$$
\chi_{\square ; \square \otimes \square}=\frac{1}{2}\left[\operatorname{Tr}(X)^{2} \operatorname{Tr}(Y)+\operatorname{Tr}\left(X^{2}\right) \operatorname{Tr}(Y)+2 \operatorname{Tr}(X Y) \operatorname{Tr}(X)+2 \operatorname{Tr}\left(X^{2} Y\right)\right],
$$




$$
\chi_{\mathrm{B}_{\mathrm{B} \otimes \square}}=\frac{1}{2}\left[\operatorname{Tr}(X)^{2} \operatorname{Tr}(Y)-\operatorname{Tr}\left(X^{2}\right) \operatorname{Tr}(Y)-2 \operatorname{Tr}(X Y) \operatorname{Tr}(X)+2 \operatorname{Tr}\left(X^{2} Y\right)\right] .
$$

For these two restricted Schur polynomials we again do not need a projector to implement the restricted trace. If we take

$$
\chi_{R, R_{\alpha}}=\frac{1}{2 !} \sum_{\sigma \in S_{3}} \operatorname{Tr}_{R_{\alpha}}\left(\Gamma_{R}(\sigma)\right) X_{i_{\sigma(1)}}^{i_{1}} X_{i_{\sigma(2)}}^{i_{2}} Y_{i_{\sigma(3)}}^{i_{3}},
$$

then $R_{\alpha}$ is an irreducible representation of $S_{2} \times S_{1}$. The $S_{2}$ subgroup is obtained by taking those elements of $S_{3}$ that act on the indices of the $X$ s, i.e. $\{1,(12)\}$. To compute

$$
\chi_{\Psi_{; \square \otimes \square}}=\frac{1}{2}\left[\operatorname{Tr}(X)^{2} \operatorname{Tr}(Y)+\operatorname{Tr}\left(X^{2}\right) \operatorname{Tr}(Y)-\operatorname{Tr}(X Y) \operatorname{Tr}(X)-\operatorname{Tr}\left(X^{2} Y\right)\right] ，
$$

we used the projector

$$
P_{\bigoplus_{\rightarrow \square^{\otimes}}}=\frac{1}{2}\left(1+\Gamma_{\boxplus}((12))\right) .
$$

To compute

$$
\chi_{\Psi_{; \mathrm{B}{ }_{\square}}}=\frac{1}{2}\left[\operatorname{Tr}(X)^{2} \operatorname{Tr}(Y)-\operatorname{Tr}\left(X^{2}\right) \operatorname{Tr}(Y)+\operatorname{Tr}(X Y) \operatorname{Tr}(X)-\operatorname{Tr}\left(X^{2} Y\right)\right],
$$

we used the projector

$$
P_{\Psi_{\rightarrow} \square_{\square}}=\frac{1}{2}\left(1-\Gamma_{\boxplus}((12))\right)
$$

For more details on these projectors see appendix B.2 of [5] and appendix A of [7]. Comparing these expressions to the expressions in appendix E.2 of [10], it is clear that the basis furnished by the restricted Schur polynomials does not coincide with the basis of $[10]$.

We can use the $\Sigma$ map of [9] to construct new operators built out of $Z$ and $Z^{*}$. Under the map $\Sigma, B_{\alpha}=\Sigma^{-1}\left(O_{\alpha}\right)$ becomes a sum over elements of the Brauer algebra. In [9] it was argued that if $\gamma O_{\alpha} \gamma^{-1}=O_{\alpha}$ for $\gamma \in S_{n} \times S_{m}$ then $\gamma B_{\alpha} \gamma^{-1}=B_{\alpha}$ for $\gamma \in S_{n} \times S_{m}$. Also, again using a result of [9], $\left(\operatorname{Tr}_{m+n}\right.$ denotes a trace over $V^{\otimes(n+m)}$ and $\operatorname{Tr}_{m, n}$ denotes a trace over $V^{\otimes n} \otimes \bar{V}^{\otimes m}$ )

$$
\operatorname{Tr}_{m, n}\left(B_{\alpha} B_{\beta}\right)=\operatorname{Tr}_{m+n}\left(O_{\alpha} O_{\beta}\right)=\frac{\mathcal{N}_{\alpha}}{n ! m !} \delta_{\alpha \beta} .
$$

Thus, the operators

$$
\eta_{\alpha}=\operatorname{Tr}_{m, n}\left(B_{\alpha} Z^{\otimes n} \otimes Z^{* \otimes m}\right),
$$

have a diagonal two point function

$$
\left\langle\eta_{\alpha} \eta_{\beta}^{\dagger}\right\rangle=\mathcal{N}_{\alpha} \delta_{\alpha \beta}
$$


For $m=n=1$ we find

$$
\begin{aligned}
& B_{1}=\Sigma\left(\frac{1}{2}(1+(12))\right)=\frac{1}{2}\left(1+C_{1 \overline{1}}\right), \\
& B_{2}=\Sigma\left(\frac{1}{2}(1-(12))\right)=\frac{1}{2}\left(1-C_{1 \overline{1}}\right) .
\end{aligned}
$$

These do not match the operators given in appendix A.4.1 of [9], implying that the restricted Schur polynomials do not coincide with the basis constructed in [9] either. This is clear when one notes that the coefficients on the projectors in [9] are $N$ dependent; there is no way in which our operators could pick up $N$ dependent coefficients.

Recall that weights are assigned to boxes in a Young diagram by assigning $N$ to the box in the upper left hand corner of the Young diagram, adding one each time we move to the right and subtracting one each time we move down. Thus, box $i$ in the Young diagram

has weight $c_{i}$ with $c_{1}=c_{5}=N, c_{2}=N+1, c_{3}=N+2$ and $c_{4}=N-1 . f_{R}$ is the product of weights of the Young diagram, so that, for example

$$
f_{\square}=N^{2}\left(N^{2}-1\right)(N+2) \text {. }
$$

Next, since

$$
\text { hooks }(\square \square \square)=5 \text { ! hooks }(\square \square)=3 \text { ! hooks }(\square)=2 \text { !, }
$$

we have from $(2.2)$

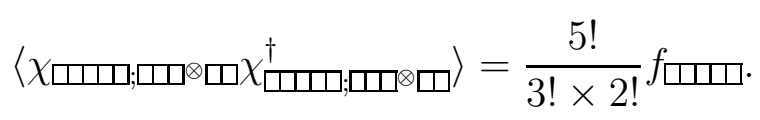

Similary,

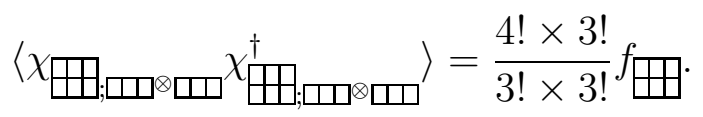

If any of the labels on the restricted Schur polynomial do not match, the correlator vanishes

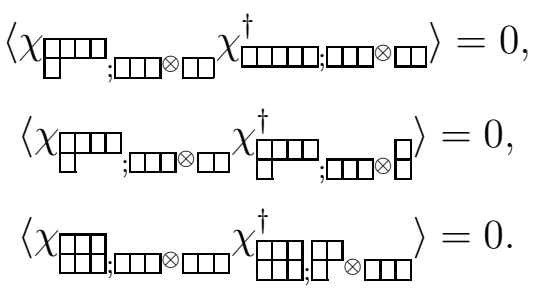


To determine which $S_{n} \times S_{m}$ irreducible representations are subduced by a particular $S_{n+m}$ irreducibe representation is easy: assume that the Young diagram $R$ describes the irreducible representation of $S_{n+m}$ that we are studying. Consider all possible ways of removing $n$ boxes from $R$ so that the remaining $m$ boxes form a legal Young diagram $R_{m}$. Remove the $n$ boxes preserving common sides and take the tensor product of the removed pieces to get $R_{n}$. This rule is easily illustrated with an example; consider

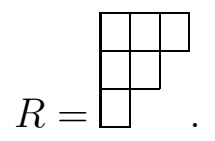

Assume that $n=m=3$. Denoting removed boxes with an $x$ we have

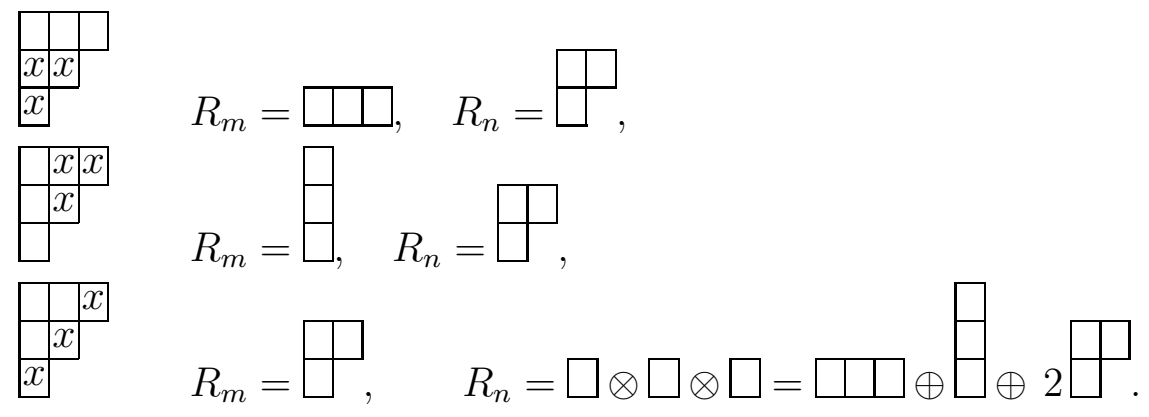

Thus, $R$ subduces 6 irreducible representations of $S_{3} \times S_{3}$.

\section{Generalization to Multi-Matrix Models}

The above results generalize in a simple way to multi-matrix models. Consider a model of $M$ matrices and assume that $\chi_{R, R_{\alpha}}$ is built from $m_{i}$ matrices of each type. Then $R_{\alpha}$ is an irreducible representation of $S_{m_{1}} \times S_{m_{2}} \times \cdots \times S_{m_{M}}$. To remove self contractions (present if we have real matrices or if we build $\chi_{R, R_{\alpha}}$ from complex matrices and their adjoints) we simply normal order $\chi_{R, R_{\alpha}}$. This gives a unified treatment of both branes/antibrane systems and operators built from more than one Higgs field. These operators are labeled by $M+1$ Young diagrams, one with $m_{1}+m_{2}+\ldots+m_{M}$ boxes, $R$ and $M$ with $m_{i}$ boxes, $R_{i}$. In this more general case we still have $(2.2)$ with

$$
(\text { hooks })_{R_{\alpha}}=\prod_{i=1}^{M}(\text { hooks })_{R_{i}} .
$$

It is straight forward to replace boxes in the $R_{i}$ by open strings so that excited operators can be constructed and studied using the techniques developed in [5], [6], [7]. 
We again claim that the total number of restricted Schur polynomials that can be defined will be equal to the number of gauge invariant operators that can be constructed. There are some non-trivial tests we can perform of this claim. For example, consider operators built using one of each of the $M$ types. In this case, we need to start with an irreducible representation of $S_{M}$ and count how many restricted Schur polynomials we can form when the representation of the restriction is $S_{1} \times S_{1} \times \cdots \times S_{1}$ (there are $M$ factors). To get the number of irreducible representations that can be subduced from a given Young diagram $R$, we need to count the number of ways we can pull boxes off $R$ such that at each step we have a legal Young diagram. This is obviously $d_{R}$, the dimension of the $S_{M}$ representation labeled by $R$. Any of these subduced representations may be twisted, so that we obtain a total of $d_{R}^{2}$ operators. Thus, the total number of restricted Schur polynomials, found by summing over all $S_{M}$ irreducible representations, is simply

$$
\sum_{R}\left(d_{R}\right)^{2}=M !
$$

Lets now compare this to the counting of the gauge invariant operators. According to Polya theory, the number of gauge invariant operators is given by the coefficient of $x_{1} x_{2} \cdots x_{M}$ in the expansion of

$$
\prod_{k=1}^{\infty} \frac{1}{1-\left(x_{1}^{k}+x_{2}^{k}+\cdots+x_{M}^{k}\right)}=\sum_{n_{1}, n_{2}, \cdots, n_{m}} t\left(n_{1}, n_{2}, \cdots, n_{M}\right) x_{1}^{n_{1}} x_{2}^{n_{2}} \cdots x_{M}^{n_{M}} .
$$

It is simple to see that

$$
t(1,1, \cdots, 1)=M !
$$

which supports our claim.

\section{Numerical Tests}

We have counted the number of restricted Schur polynomials $\chi_{R, R_{\alpha}}$ that be obtained when $R$ is an irreducible representation of $S_{n}$ with $n \leq 6$ and we have a total of $M=6$ matrices. In all of these cases, the number of restricted Schur polynomials equals the number of gauge invariant operators counted using Polya theory. Further, we have numerically evaluated the two point functions of these restricted Schur polynomials and verified that $(2.2)$ is indeed correct. In performing these checks, the restricted characters $\operatorname{Tr}_{R_{\alpha}}\left(\Gamma_{R}[\sigma]\right)$ were evaluated by explicitly constructing the matrices $\Gamma_{R}[\sigma]$. Each representation used was obtained by induction. One induces a reducible representation; the irreducible representation required was isolated using projection operators 
built from the Casimir obtained by summing over all two cycles. The restricted trace was then evaluated with the help of suitable projectors. See appendix B.2 of [5] and appendix A of [7] for more details. In all cases the numerical result is in exact agreement with $(2.2)$.

\section{Conclusions}

Restricted Schur polynomials provide a useful parameterization of the complete set of gauge invariant variables of multi-matrix models. They have diagonal two point functions. Since in the labeling of the restricted Schur polynomial, each type of matrix has its own Young diagram, the technology for attaching open strings has a straight forward generalization to the operators considered in this article.

For brane-anti-brane operators, the restricted Schur polynomials do not coincide with the Brauer basis constructed in [9]. Since the Brauer projectors are $N$ dependent, the relation between the two bases is $N$ dependent. It seems that the Brauer basis may be the most useful for identifying brane - anti-brane operators and the restricted Schur polynomial basis for stringy excitations. It is plausible that there is a simple relation between the restricted Schur polynomials and the operators of [10]. For example, $\chi_{\Psi_{; \square^{\otimes}}}-\chi_{\square_{;: \square_{\square}}}$ is (up to an overall constant factor) equal to the operators constructed in E.2 and E.3 of [10]. Since the restricted Schur polynomials have an interpretation in terms of attaching open strings, developing this relation may well shed light on the interpretation of the labels of the operators constructed in [10]. We leave this interesting problem for the future.

Finally, it would be interesting to explore finite $N$ effects. These effects are encoded in the fact that our Young diagram labels can have at most $N$ rows. Specifically, in the restricted Schur polynomial $\chi_{R,\left(r_{\alpha_{1}}, r_{\alpha_{2}}\right)}$ we must require that the Young diagram $R$ has at most $N$ rows; the same will automatically be true for $r_{\alpha_{1}}$ and $r_{\alpha_{2}}$. This should translate into a generalization of the stringy exclusion principle present for Schur polynomials built using a single matrix $Z$. Finite $N$ counting for multi-matrix operators has been considered in [10],[14]. For example, the number of operators built using $\mu_{1} X$ fields and $\mu_{2} Y$ fields, at infinite $N$ is given by $^{2}$

$$
N\left(\mu_{1}, \mu_{2}\right)=\sum_{T} \sum_{\Lambda} C(T, T, \Lambda) g(\mu ; \Lambda)
$$

In this formula, $T$ is a representation of $S_{n}$ with $n=\mu_{1}+\mu_{2}, C(T, T, \Lambda)$ is the coefficient of $\Lambda$ in the (inner) tensor product $T \otimes T$ and $g(\mu ; \Lambda)$ is the Littlewood-Richardson

\footnotetext{
${ }^{2}$ We are considering the case of two matrices for simplicity. The formula for $M$ matrices has been determined in [10],[14].
} 
coefficient which counts states in the representation $\Lambda$ that have the field content $\mu=$ $\left[\mu_{1}\right] \otimes\left[\mu_{2}\right]$. To get the finite $N$ counting, one simply truncates the sum over $T$ to Young diagrams with at most $N$ rows. We can see, in some simple examples, that our cut off on $R$ does indeed match the finite $N$ counting of [10],[14]. Consider for example the operators built using $3 X$ fields and a single $Y$ field. The relevant LittlewoodRichardson coefficients are

$$
g(\text { س, ㅁㅁ })=1, \quad g(\text { س, ; }
$$

The relevant inner products are

سथவ =யா,

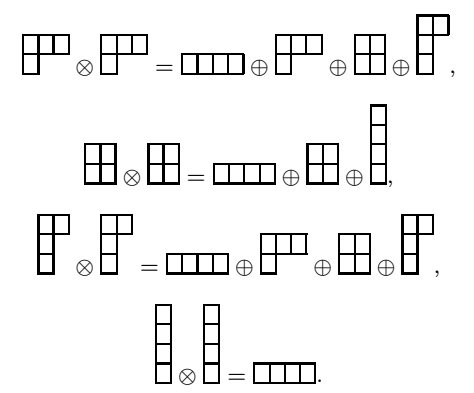

Clearly then, at infinite $N$, the number of operators we can construct is

$$
\begin{aligned}
& N(2,1)=\sum_{T}\left(C(T, T, \text { س })+C\left(T, T, \Psi^{\square}\right)\right)
\end{aligned}
$$

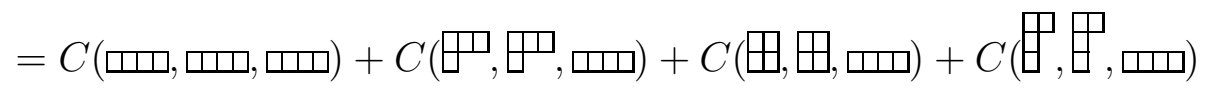

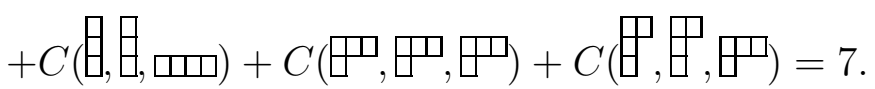

At $N=2$, this counting becomes

$$
\begin{aligned}
& N(2,1)=\sum_{T}\left(C(T, T, \text { س })+C\left(T, T, \Psi^{\boxplus}\right)\right)
\end{aligned}
$$

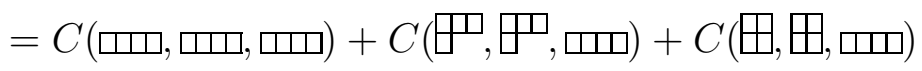

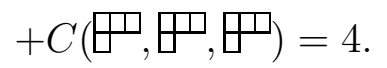

Lets now count the restricted Schur polynomials. At infinite $N$ we find 7 possible operator, with $R, r_{\alpha_{1}} r_{\alpha_{2}}$ labels given by

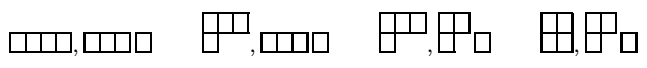

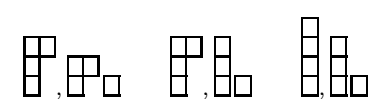


At $N=2$ there are only 4 operators, with labels given by

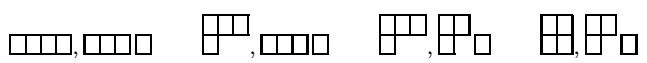

Providing a proof that our cut off on $R$ matches the finite $N$ counting of [10],[14] remains an interesting open problem.

Acknowledgements: We would like to thank Norman Ives, Sanjaye Ramgoolam and Alex Welte for enjoyable, helpful discussions and/or correspondence. We would also like to thank Sanjaye Ramgoolam for a careful reading of the manuscript and Tom Brown for useful correspondence on the first version of this article. Finally, we would like to thank Norman Ives for again pointing out which is our left hand and which is our right hand. This work is based upon research supported by the South African Research Chairs Initiative of the Department of Science and Technology and National Research Foundation. Any opinion, findings and conclusions or recommendations expressed in this material are those of the authors and therefore the NRF and DST do not accept any liability with regard thereto. This work is also supported by NRF grant number Gun 2047219.

\section{References}

[1] J. M. Maldacena, "The large N limit of superconformal field theories and supergravity," Adv. Theor. Math. Phys. 2, 231 (1998) [Int. J. Theor. Phys. 38, 1113 (1999)] [arXiv:hep-th/9711200];

S. S. Gubser, I. R. Klebanov and A. M. Polyakov, "Gauge theory correlators from non-critical string theory," Phys. Lett. B 428, 105 (1998) [arXiv:hep-th/9802109];

E. Witten, "Anti-de Sitter space and holography," Adv. Theor. Math. Phys. 2, 253 (1998) [arXiv:hep-th/9802150].

[2] A. Jevicki and B. Sakita, "The quantum collective field method and its application to the planar limit," Nucl. Phys. B 165, 511 (1980);

A. Jevicki and B. Sakita, "Collective Field Approach To The Large N Limit: Euclidean Field Theories," Nucl. Phys. B 185, 89 (1981);

S. R. Das and A. Jevicki, "String Field Theory and Physical Interpretation of D = 1 Strings," Mod. Phys. Lett. A 5, 1639 (1990).

[3] S. Corley, A. Jevicki and S. Ramgoolam, "Exact correlators of giant gravitons from dual N = 4 SYM theory," Adv. Theor. Math. Phys. 5, 809 (2002) [arXiv:hep-th/0111222].

[4] V. Balasubramanian, D. Berenstein, B. Feng and M. x. Huang, "D-branes in Yang-Mills theory and emergent gauge symmetry," JHEP 0503, 006 (2005) [arXiv:hep-th/0411205]. 
[5] R. de Mello Koch, J. Smolic and M. Smolic, "Giant Gravitons - with Strings Attached (I)," JHEP 0706, 074 (2007), arXiv:hep-th/0701066.

[6] R. de Mello Koch, J. Smolic and M. Smolic, "Giant Gravitons - with Strings Attached (II)," JHEP 0709049 (2007), arXiv:hep-th/0701067.

[7] D. Bekker, R. de Mello Koch and M. Stephanou, "Giant Gravitons - with Strings Attached (III)," arXiv:0710.5372 [hep-th].

[8] R. de Mello Koch and R. Gwyn, "Giant graviton correlators from dual SU(N) super Yang-Mills theory," JHEP 0411, 081 (2004) [arXiv:hep-th/0410236];

T. W. Brown, "Half-BPS SU(N) correlators in N = 4 SYM," arXiv:hep-th/0703202;

S. Ramgoolam, "Wilson loops in 2-D Yang-Mills: Euler characters and loop equations," Int. J. Mod. Phys. A 11, 3885 (1996) [arXiv:hep-th/9412110];

Y. Hatsuda and Y. Matsuo, "Symmetry and integrability of non-singlet sectors in matrix quantum mechanics," J. Phys. A 40, 1633 (2007) [arXiv:hep-th/0607052].

[9] Y. Kimura and S. Ramgoolam, "Branes, Anti-Branes and Brauer Algebras in Gauge-Gravity duality," arXiv:0709.2158 [hep-th].

[10] T. W. Brown, P. J. Heslop and S. Ramgoolam, "Diagonal multi-matrix correlators and BPS operators in N=4 SYM," arXiv:0711.0176 [hep-th].

[11] T. Brown, R. de Mello Koch, S. Ramgoolam and N. Toumbas, "Correlators, probabilities and topologies in N = 4 SYM," JHEP 0703, 072 (2007) [arXiv:hep-th/0611290].

[12] T. W. Brown, "Permutations and the Loop," arXiv:0801.2094 [hep-th].

[13] S. Corley and S. Ramgoolam, "Finite factorization equations and sum rules for BPS correlators in N = 4 SYM theory," Nucl. Phys. B 641, 131 (2002) [arXiv:hep-th/0205221].

[14] F. A. Dolan, "Counting BPS operators in N=4 SYM," Nucl. Phys. B 790, 432 (2008) [arXiv:0704.1038 [hep-th]]. 